\title{
THE CONNECTION OF FINE-STRUCTURE PHOTOSPHERIC FEATURES IN ACTIVE REGIONS WITH MAGNETIC FIELDS
}

\author{
N.V. STESHENKo \\ (Crimean Astrophysical Observatory, \\ Nauchny, Crimea, U.S.S.R.)
}

1. The fine structure of the proton sunspot group of July 4-8, 1966 was studied on the basis of high-resolution heliograms. The comparison of the orientation between penumbral filaments and the transverse magnetic fields (observed by A. B. Severny and T.T. Tsap) shows that the direction of the filaments coincides in general with that of the magnetic field.

2. Measurements of the magnetic fields of smallest pores $\left(1 \cdot 5^{\prime \prime}-2^{\prime \prime}\right)$ showed that the pores are always connected with strong magnetic field (in average 1400 gauss), which is localized at the same small area as the pore.

3. Magnetic fields of faculae are concentrated in small elements with the dimension not exceeding $1 \cdot 5^{\prime \prime}-3^{\prime \prime}$. Magnetic-field strength $H_{\|}$of about $45 \%$ of facular granules is within the limits of photographic measuring errors (approximately 25 gauss). For a quarter of all facular granules the strength $H_{\|}$is from $25-50$ gauss; about $30 \%$ of facular granules have $H_{\|}>50$ gauss, and sometimes there appear faculae with field strength of about 200 gauss. The magnetic-field strength of facular granules, which are found directly above spots, is 10-20 times less than the field strength of spots. This field is $80-210$ gauss only.

4. All observational data mentioned above show that the appearance of the finestructure features in active regions is directly connected with the fine structure of magnetic field of different strength and different orientation. The study of highresolution heliograms gives additional information about the fine structure of the magnetic field.

\section{DISCUSSION}

McIntosh: Your slide of the sunspot group on July 6, 1966 showed penumbral structure between the strong spots aligned North to South and parallel to the magnetic-field lines. I have observations later that same day that show the penumbral filaments at the same location then oriented East to West. Did your magnetic observations show a corresponding change in the orientation of magnetic lines of force?

Steshenko: Observations on July 7 show the field orientation as East to West in that location. 